\title{
Aurothiolates enhance the replication of Semliki Forest virus in the CNS and the exocrine pancreas
}

\author{
Martina F Scallan ${ }^{1}$ and John K Fazakerley*,2 \\ ${ }^{1}$ Department of Microbiology, University College Cork, National University of Ireland $;{ }^{2}$ Virus Research Group, University \\ of Edinburgh, EH9 1QH, UK
}

\begin{abstract}
The A7(74) strain of Semliki Forest virus (SFV) is avirulent and the L10 strain virulent in adult mice. A7(74) infection of adult mouse brain gives rise to small discrete foci of infection which, in immunocompetent animals, are cleared within 10 days. In contrast $L 10$ infection results in a widespread and fatal central nervous system infection. Aurothiolates are linear, 2-coordinate complexes in which two ligands are covalently bound on either side of a gold nucleus in a +1 oxidation state (gold (I)). Pretreatment of A7(74) infected mice with two distinct aurothiolates (sodium aurothiomalate and aurothioglucose) resulted in significantly increased brain virus titers, and large confluent areas of infection in the brain similar to the pattern of infection seen with the L10 strain. The gold (I) moiety of aurothiolates was demonstrated to be the active component, since thiomalic acid when administered alone had no potentiating effect on the infection. Although both aurothiolates allowed productive replication and spread of A7(74) within the nature mouse brain, enhanced neuronal destruction was not apparent. There were no significant changes in virus distribution in any other tissue except for the exocrine pancreas and the myocardium where widespread infection of the acinar cells and occasional infected myocytes were observed.
\end{abstract}

Keywords: alphavirus; Semliki Forest virus; gold; neurovirulence; neurovirology

\section{Introduction}

Semliki Forest virus (SFV) is a well characterized neurotropic alphavirus. A number of strains, differing in their virulence for mice have been isolated from mosquitoes. Amongst these is a virulent L10 strain, isolated from a pool of Aedes abnormalis mosquitoes captured in Uganda (Smithburn and Hadow, 1944) and an avirulent strain, A7(74), derived from Aedes argenteopunctatus mosquitoes caught in Mozambique (McIntosh et al, 1961; Bradish et al, 1971). Following intraperitoneal (ip) inoculation into 3-4-week-old mice, L10 produces a lethal encephalitis which results in death within 6 days. In contrast A7(74) causes a subclinical encephalitis characterized by restricted virus replication and survival of infected central nervous system (CNS) neurons (Fazakerley et al, 1993; Amor et al, 1996). Both humoural and cell-mediated immune responses contribute to the clearance of

*Correspondence: J Fazakerley, Virus Research Group, University of Edinburgh, Summerhall, Edinburgh EH9 1QP

Received 3 December 1998; revised 3 March 1999; accepted 13 April 1999
A7(74) from the CNS, but do not account for the focal nature of this infection, since the restricted pattern of infection is also observed in adult mice with severe combined immunodeficiency (SCID) (Fazakerley et al, 1993; Amor et al, 1996). Clearance of A7(74) in immunocompetent mice coincides with the appearance of temporary lesions of CD8 T-cell mediated demyelination within the CNS (Suckling et al, 1978; Fazakerley and Webb, 1987a,b; SubakSharpe et al, 1993).

Both strains of SFV have been reported to replicate to similar titers in extraneural tissues, predominantly muscle (Pusztai et al, 1971; Amor et al, 1996), producing a plasma viraemia which delivers virus to the CNS. In immunocompetent adult Balb/c mice, foci of A7(74) replication in peripheral tissues are difficult to find by immunostaining or in situ hybridization, but focal infection of skeletal muscle fibres has been observed in infected SCID mice (Amor et al, 1996). SFV is thought to enter the CNS by passage across cerebral vascular endothelial cells (Pathak and Webb, 1974, 1980). 
Once within the CNS of adult mice, L10 and A7(74) display very different abilities to replicate and spread (Fazakerley et al, 1993). In both instances virus first appears as small foci of infected cells around cerebral capillaries. In the L10 infection virus spreads rapidly from these initial foci to infect large areas throughout the brain. With A7(74) little or no spread occurs from the original perivascular foci. In contrast to this focal CNS infection in 3-4-week-old mice, infection of mice with $A 7(74)$ at 12 days of age or less, results in widespread fatal encephalitis (Fazakerley et al, 1993; Oliver et al, 1997). This age-related change in virulence is not due to maturity of specific immune responses since infection of 3-4-weekold SCID mice remains focal (Fazakerley et al, 1993). The inability of A7(74) to spread in the brains of 3-4-week-old mice has been linked to a restriction of virus replication which is dependent upon the differentiation state of CNS cells (Pathak and Webb, 1978; Fazakerley et al, 1993; Oliver et al, 1997; Oliver and Fazakerley, 1998). Despite extensive examination of infected brain tissue under the electron microscope, neither virus particles nor discrete nucleocapsids are observed in CNS cells of adult mice infected with A7(74) (Pathak and Webb, 1988a). Amorphous and fibrous viral material, described as viral core aggregates (VCA), accumulates in isolated CNS cells (Pathak and Webb, 1988b; Fazakerley et al, 1993).

It has been demonstrated that treatment of 3-4week-old mice with the gold compound sodium aurothiomalate (GSTM) prior to inoculation with A7(74) renders this infection lethal (Allner et al, 1974; Bradish et al, 1975; Pathak and Webb, 1983; Mehta and Webb, 1982, 1990, Gates et al, 1984; Mehta et al, 1987, 1990). GSTM is an aurothiolate compound which has been used in the treatment of rheumatoid arthritis. Aurothiolates are linear, 2coordinate complexes in which two ligands are covalently bound on either side of a gold nucleus in a +1 oxidation state (gold (I)). Early studies suggested these gold compound affected the outcome of SFV infection by an effect on macrophages (Allner et al, 1974; Bradish et al, 1975). Subsequent studies confirmed that these compound have an effect on macrophages (Mehta and Webb, 1982, 1990) but suggested that this was not the basis for their effect on the course of SFV infection (Mehta and Webb, 1987; Mehta et al, 1990). Electron microscopic studies of CNS cells from mice inoculated ip with GSTM have revealed intracellular deposits, with electron-dense profiles characteristic of soluble gold salts, confirming that the drug enters the CNS (Pathak and Webb, 1983). It has been reported that in the presence of GSTM the restriction on $A 7(74)$ virion formation in the mature CNS is relieved (Pathak and Webb, 1983). In this study we investigate the effect of two aurothiolates, sodium aurothiomalate (GSTM) and aurothioglu- cose (GSTG) on the pathogenesis of A7(74) infection, and show that increased virulence is associated with enhanced replication and spread of virus in the brain and pancreas. We demonstrate that it is the gold (I) moiety in these compounds which is active in this respect and that aurothiolateenhanced replication of virus in the acinar cells of the exocrine pancreas is associated with apoptotic cell death, but the widespread infection of neurons is not.

\section{Results}

Mortality

Forty-two female Balb/c mice (3-4-weeks old) were divided into six groups of seven mice. Two groups were administered $10 \mathrm{mg}$ of GSTM (ip), two groups $10 \mathrm{mg}$ of GSTG (ip). Two groups received no pretreatment. Four hours later one of each of the GSTM and GSTG pre-treated groups was inoculated (ip) with $5 \times 10^{3}$ p.f.u. of $A 7(74)$. At the same time the two untreated groups were inoculated (ip), one with $5 \times 10^{3}$ p.f.u. of L10 and the other with the same quantity of A7(74). The mice were observed daily over the course of 2 weeks for signs of disease. Figure 1 shows the survival of the various groups.

All mice infected with the L10 strain of SFV were dead by 6 days post infection. In contrast, all mice infected with the A7(74) strain survived and no clinical signs were observed over the 2-week observation period. Mice administered GSTM and infected with A7(74) showed signs of hind limb

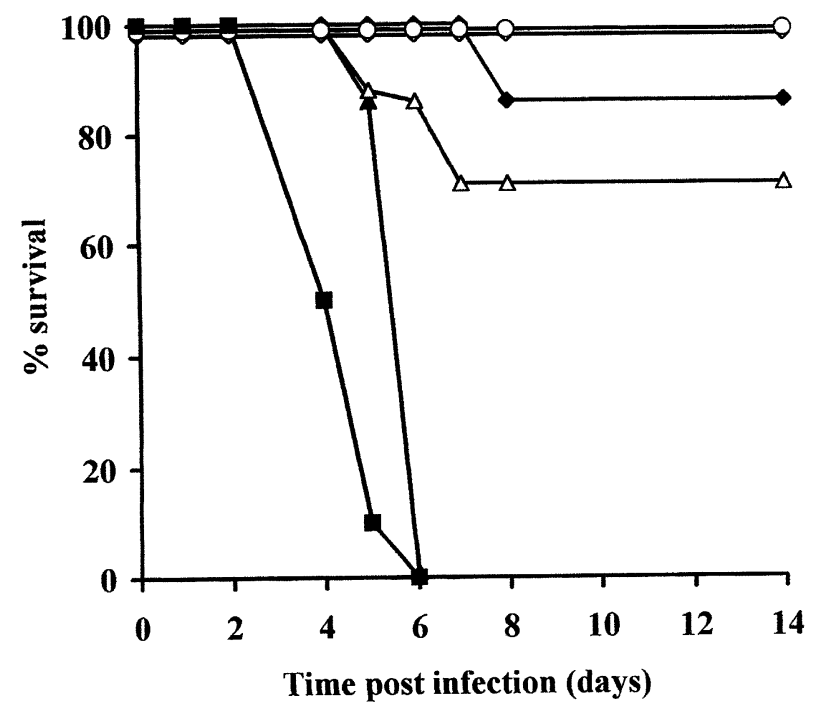

Figure 1 Survival curves for 3-4-week-old Balb/c mice inoculated ip with $5 \times 10^{3}$ p.f.u. of either the A7(74) or L10 strain of Semliki Forest virus. $\bigcirc$ A7(74) infected mice; $\square$ L10 infected mice; $\boldsymbol{A} A 7(74)$ infected mice pre-treated with $10 \mathrm{mg}$ GSTM ip; $\triangle$ A7(74) infected mice pre-treated with $10 \mathrm{mg}$ GSTG ip; $\diamond$ control mice administered $10 \mathrm{mg}$ of GSTM ip; $\diamond$ control mice administered $10 \mathrm{mg}$ of GSTG ip. Each group contained a minimum of seven mice. 
paralysis by post infection day (PID) 4 and were all dead by PID 6. Clearly this compound renders the normally avirulent A7(74) infection virulent. Mice administered $10 \mathrm{mg}$ of GSTM alone, displayed ruffled fur from 3 days and were hunched in appearance during days 6 and 7, one died 8 days after inoculation but the remaining mice survived. Amongst the seven mice administered GSTG and infected with A7(74) one died on PID 5 and another on PID 7, the remaining five survived (overall mortality $29 \%$ ). In subsequent experiments overall mortality with A7(74) plus GSTG averaged 44\% (7/ 16). All of the mice administered GSTG alone maintained a healthy appearance.

To investigate the effect of aurothiolates on the CNS infection a second study was undertaken. Five groups, each comprising $12-18 \mathrm{BALB} / \mathrm{c}$ mice (3-4 weeks old), were inoculated with $5 \times 10^{3}$ p.f.u. of SFV. One group was infected with the L10 strain, the remaining four groups with A7(74). Two of the A7(74) infected groups had been administered 10 mg doses of GSTM or GSTG $4 \mathrm{~h}$ previously. A further group was inoculated with the thiomalic acid (TMA) component of GSTM prior to infection with A7(74), and the fourth group received A7(74) alone. Three mice were sampled from each group at $24 \mathrm{~h}$ intervals. For each mouse, the virus titer was determined in one half of the brain by plaque assay and the distribution of virus was determined in the other half by in situ hybridization and autoradiography. In addition, samples of all major peripheral tissues were taken and virus distribution was determined by in situ hybridization and immunostaining.

\section{Brain virus titers}

Figure 2 shows brain virus titers in mice infected with either the L10 or A7(74) strain of SFV or infected with the A7(74) strain following administration of aurothiolates. Brain virus titers rose most rapidly in L10 infected mice reaching on average $6 \times 10^{8}$ p.f.u./g by PID 4. In A7(74) infected mice, brain virus titers rose more slowly to a maximum of $8 \times 10^{5}$ p.f.u./g on PID 6 . By PID 3 both strains were detected in the brain of all unpre-treated mice. At this time post infection the strain-dependent difference in the levels of brain virus was most profound. Levels of brain virus were significantly higher $(P<0.02$, Student's two tailed $t$-test $)$ with L10 than with A7(74), with average titers of $4 \times 10^{8}$ p.f.u./g and $1.7 \times 10^{2}$ p.f.u./g, respectively. A7(74) was eventually cleared from the brain, with only 1 of 3 mice harbouring detectable virus on PID 9. No virus was detected in the brain of $A 7(74)$ infected mice by plaque titration on PID 14 (data not shown).

Pre-treatment of 3-4-week-old mice with GSTM or GSTG increased the A7(74) brain virus load by up to 30 000-fold. On PID 4 levels of A7(74) in the brain reached on average $2.9 \times 10^{3}$ p.f.u./g in untreated mice, $2 \times 10^{7}$ p.f.u./g in GSTM treated mice and
$1 \times 10^{8}$ p.f.u./g in mice pre-treated with GSTG. This was a significant enhancement of virus replication $(P<0.02$ in both cases by two tailed Student's $t$-test). On days 2, 3 and 4 the GSTG group titers were significantly higher $(P<0.03)$ than the GSTM group. Brain virus titers continued to rise in mice administered GSTM up to PID 5. By PID 6 all of the GSTM pre-treated mice were dead.

\section{Central nervous system spread}

Figure 3 shows the distribution of SFV RNA in representative brain sections from each of the groups of mice. As reported previously (Fazakerley et al, 1993), small, randomly distributed foci of viral RNA-positive cells are characteristic of A7(74) infection in the mature brain. This contrasts with widespread virus-positivity observed in L10 infected mice by PID 5 (Fazakerley et al, 1993), and with the widespread nature of A7(74) infection in the CNS of neonatal mice (Oliver et al, 1997). Pretreatment with the TMA component of GSTM

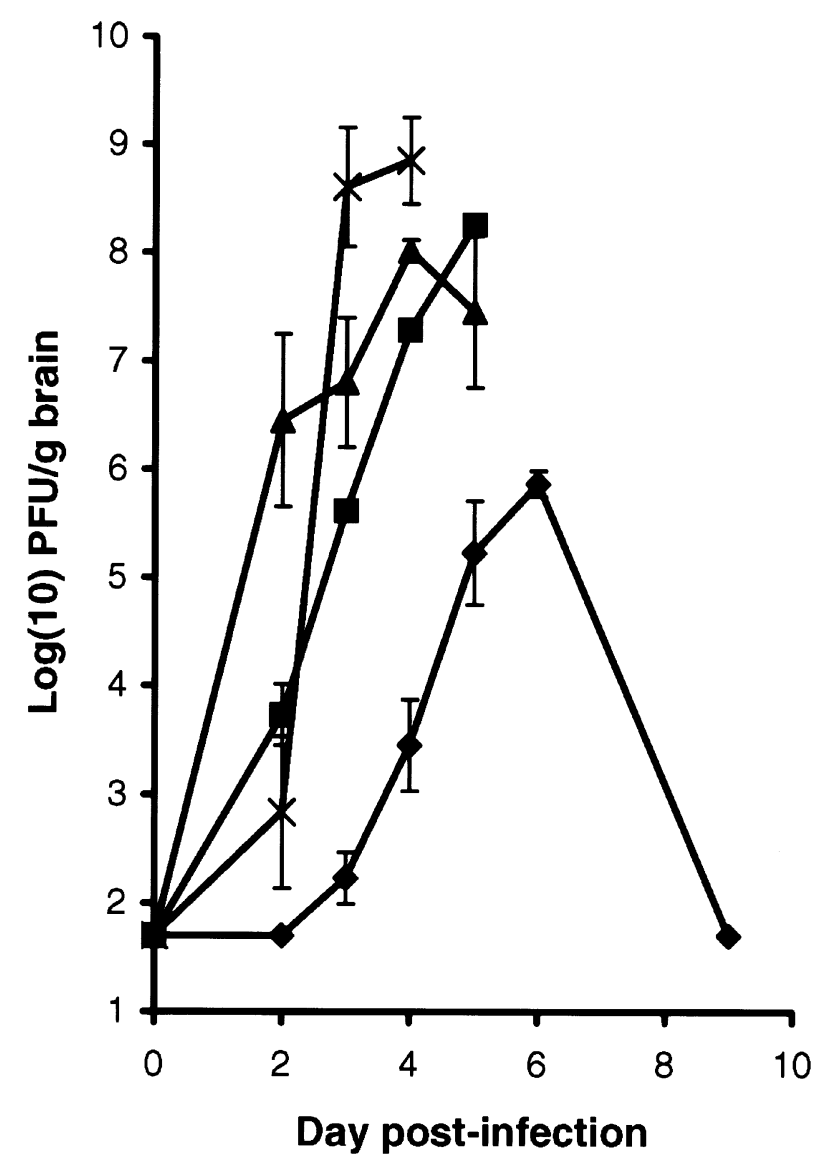

Figure 2 Brain virus titers in Balb/c mice inoculated ip with $5 \times 10^{3}$ p.f.u. of SFV L10 (X) or SFV A7(74): unpre-treated mice $(\diamond)$; mice pre-treated with $10 \mathrm{mg}$ of GSTM $(\boldsymbol{\square})$; mice pre-treated with $10 \mathrm{mg}$ of GSTG $(\boldsymbol{\Delta})$. Symbols represent the mean of $3-4$ individual mice. The limit of detection of the assay was $10^{1,7}$ p.f.u./g. The error bars show one standard deviation above and below the mean. 


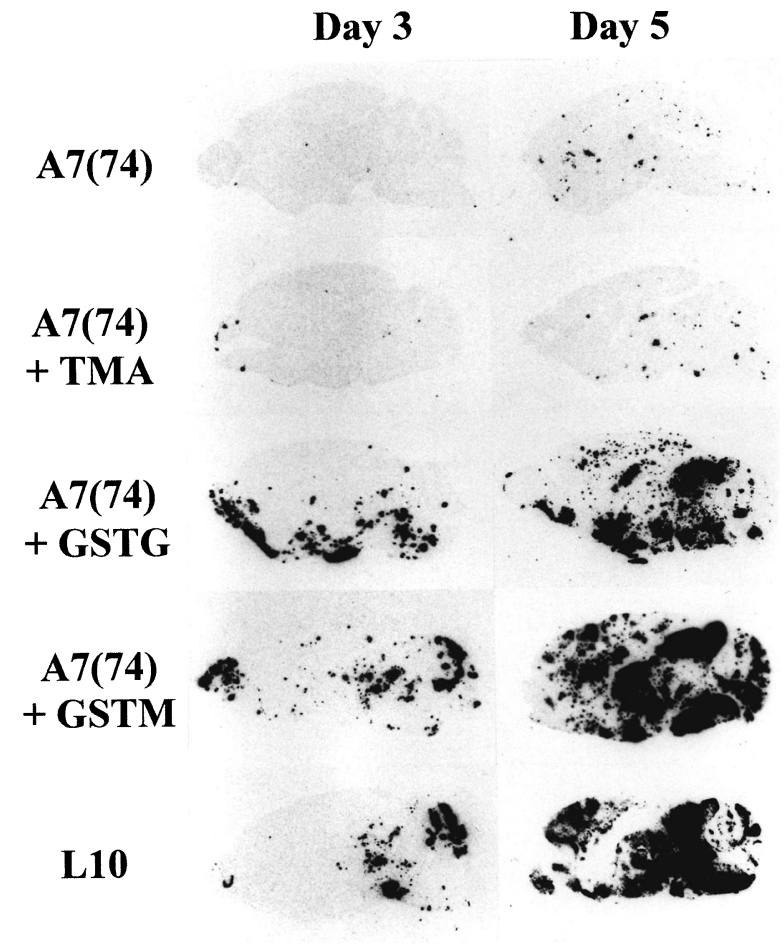

Figure 3 Representative autoradiographic images of sagittal sections of brains from 3-4-week old Balb/c mice demonstrating the effect of aurothiolates on spread of the A7(74) strain of Semliki Forest virus in the brain. The black areas indicate viral RNA.

prior to $A 7(74)$ infection had no effect on virus spread (Figure 3). Virus-positive cells remained confined to perivascular foci. In contrast, pretreatment with either GSTM or GSTG relieved the restriction on the spread of $A 7(74)$; large confluent areas of virus-positive cells were observed in the brains of GSTG and GSTM pre-treated mice by PID 5. In line with the normal course of A7(74) CNS infection, virus first appeared in the pre-treated mice as small perivascular foci. However, in the presence of gold compounds A7(74) infection resembled L10 infection, with virus spreading rapidly throughout the brain to create large confluent areas of infection.

Brain sections were further examined for viral protein expression and apoptosis of infected cells. Figure 4 shows representative photomicrographic images of infected brain tissue immunostained for SFV structural proteins (Figure $4 \mathrm{~A}-\mathrm{D}$ ) or doublelabelled for SFV structural proteins and TUNEL positive nuclei (Figure 4D, E and H). In line with the in situ hybridization data, only very limited numbers of viral antigen positive CNS cells were observed in non-pre-treated A7(74) infected mice (Figure 4A), in contrast, widespread areas of infection, containing many viral antigen positive cells were observed in the brains of A7(74) infected mice pre-treated with GSTM (Figure 4B), and in L10 infected mice (Figure 4C). Despite, this enhancement of viral replication, there was no increase in the number of TUNEL positive nuclei in the CNS of A7(74) infected mice pre-treated with aurothiolates (Figure 4D). In aurothiomalate pre-treated and in L10 infected brain sections, neuronal processes stained positive for viral antigen, but in $A 7(74)$ infected brain tissue from non pre-treated mice only viral antigen-positive cell bodies were visible. Both A7(74) and L10 infected neurons had pale, swollen nuclei with prominent nucleoli (Figure 4B and C).

\section{Infection of peripheral tissues}

Random sections of peripheral tissues including heart, lung, liver, gut, pancreas, spleen, thymus, lymph nodes, kidney, skeletal, smooth and cardiac muscle, glandular tissue and adrenal gland were examined for the presence of viral RNA by in situ hybridization with digoxigenin-labelled riboprobes.

Foci of L10 infection were clearly visible in skeletal muscle fibres on PID 4 (Figure 4F), in one mouse an isolated infected spleen cell was observed and in another a focus of infection in heart muscle (PID 2). All other peripheral tissues were negative for viral RNA. No evidence of SFV infection was detected in peripheral tissues taken from $A 7(74)$ infected adult mice on PID 2. However, adult mice administered GSTM or GSTG prior to infection with A7(74), had obvious peripheral sites of infection. Viral RNA was observed in myocytes in the heart (GSTM and GSTG) and rarely within cells in the spleen (GSTG) and the salivary gland (GSTG). Strikingly, pancreatic tissue from pre-treated mice was laden with viral RNA (GSTM and GSTG: see Figure 4E). No evidence of $A 7(74)$ infection was observed in any other peripheral tissue from pretreated mice, including skeletal muscle. Sections of pancreas were further examined for viral protein expression and apoptosis of infected cells (Figure $4 \mathrm{G}$ and $\mathrm{H}$ ). Viral antigen expression was observed in the acinar cells of the exocrine pancreas. Apoptotic nuclei were seen adjacent to infected cells (Figure $4 \mathrm{H})$.

'Gold' coloured deposits were observed in the kidneys of mice administered GSTM, but not in mice administered GSTG.

\section{Discussion}

Here we demonstrate that the gold (I) moiety in GSTM and GSTG potentiates A7(74) infection of CNS cells, and of acinar cells in the exocrine pancreas, since both GSTM and GSTG enhance the replication and spread of $A 7(74)$ within these organs while the thiomalic acid component of 

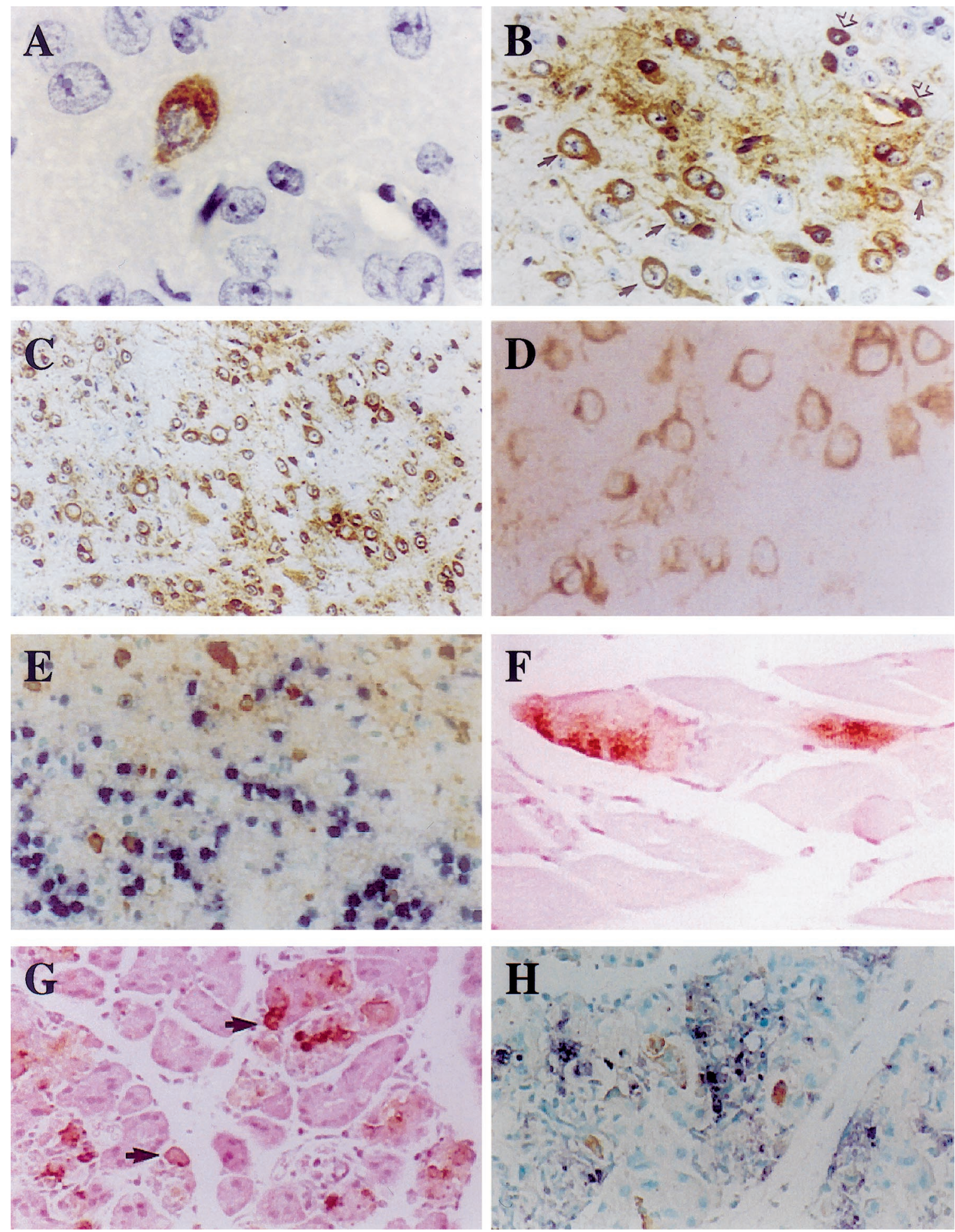

Figure 4 Photomicrographic images of infected mouse tissues. (A-D) brain sections immunostained for SFV capsid protein and counterstained with hematoxylin: (A) Single positive neuron in the hypothalamus of an SFV A7(74) infected adult mouse brain (PID 6). (B) large area of infection, including cells with distinct neuronal morphology (arrows) and intensely stained small round cells, most probably glial cells (arrow heads) in the thalamus of an SFV A7(74) infected adult mouse pre-treated with GSTM (PID 4). (C) Widespread area of SFV L10 infection in the thalamus of an adult mouse brain on PID 3. Note the positive neuronal processes in (B), (C) and (D). The sections in (D) and (E) are immunostained for viral capsid protein (brown) and TUNEL reactive nuclei (blue): (D) Cells in the occipital cortex of an SFV A7(74) infected mouse pre-treated with GSTM. The viral protein in the cytoplasm gives a ring of staining around the unstained (TUNEL-negative, non apoptotic) nuclei. There is no hematoxylin counterstain. (E) Positive control for TUNEL staining, brain tissue from the anterior olfactory nucleus of a neonatal (P4) mouse infected intra-nasally $36 \mathrm{~h}$ previously with SFV A7(74). This section has a light neutral green counterstain. (F and $\mathbf{G}$ ) positive in situ hybridization signal (digoxigenin-labelled 
GSTM by itself has no potentiating effect. GSTM when administered alone, was more toxic for mice than GSTG alone. In addition, although both compounds enhanced A7(74) replication to similar extents, mortality was lower with GSTG and A7(74). It is possible that the glucose ligand in GSTG lends to more efficient cellular uptake and better dissemination of the drug throughout the body. This would be consistent with the higher brain virus titers in GSTG treated mice on PID 4 (Figure 2) and the gold coloured deposits observed in the kidneys of GSTM but not GSTG treated mice. The exact cause of death in the gold (I) treated and A7(74) infected mice is not known. Mortality may result from an additive effect of drug and virus, but is clearly not associated with neuronal loss within the brain. Death may have resulted from neuronal dysfunction due to high virus load or to perturbation of pancreatic or cardiac function due to the altered course of the peripheral infection.

Replication of SFV is intimately associated with smooth membrane vesicles in the cytoplasm, termed cytopathic vacuoles (CPVs). These are derived from endosomes and lysosomes, and alphavirus RNA synthesis is critically dependent upon continuous lipid synthesis (Peranen et al, 1995; Perez et al, 1991; Kuge et al, 1989; Froshauer et al, 1988). Gold (I) compounds may increase yields of A7(74) in vivo by altering the normal balance of intracellular membranes in favour of SFV infection. Evidence in support of this includes the well documented association of gold with intracellular membranes, particularly lysosomal fractions (Lawson et al, 1977; Braas et al, 1986) and the demonstration that GSTM stimulates smooth membrane production in the mouse CNS (Pathak and Webb, 1983; Mehta et al, 1990). Mature, postmitotic neurons have a poorly developed system of smooth membrane vesicles. In contrast, differentiating neurons undergoing active synaptogenesis during the first 2 weeks after birth produce large quantities of smooth membrane vesicles which are transported to growing neuronal projections (Dailey and Bridgman, 1993). Cessation of this growthrelated membranogenesis may be the maturational event responsible for switching A7(74) replication in neurons from productive to restricted, and in doing so, defining the age-related virulence of this strain of SFV (Oliver et al, 1997; Oliver and Fazakerley, 1998). The observation that neuronal processes stained positive for viral antigen in aurothiolate pre-treated A7(74) infected and in
L10 infected brain sections, whereas in A7(74) infected brain sections from non pre-treated mice, only cell bodies stained positive for viral antigens (Figure 4A-C), points to a mechanism which involves enhanced intracellular transport of viral material.

Gold (I) compounds have been used therapeutically in the management of rheumatoid arthritis. The desire to understand their therapeutic effects has lead to important insights into their biological chemistry. The spectrum of biological effects is wide and fundamentally dependent on the ability of these compounds to undergo ligand-exchange reactions with thiol groups on cysteine residues in proteins. Gold (I) compounds exert effects on various components of the immune system (Griem et al, 1995; Koike et al, 1994) and the potentiation of the infection could result from an immunomodulatory effect. Our previous studies demonstrating that CNS infection with A7(74) remains restricted in adult SCID mice (Fazakerley et al, 1993; Amor et al, 1996) would argue against an effect of gold (I) on specific immune responses. Furthermore, the pattern of infection in peripheral tissues is different in gold (I) treated mice to that seen in SCID mice. However, other mechanisms including an effect on NK cells or an effect on soluble factors such as interferons, cytokines or growth factors produced by CNS cells cannot be ruled out and remain important possible mechanisms through which the aurothiolates exert their effect.

Potent effects of gold (I) compounds have been observed on transcription factors. It has been proposed that in biological systems, gold (I) has strongest affinity for cysteine (C) residues flanked by basic lysine (K) and arginine (R) residues (KCR context), which would render the DNA binding sites of transcription factors such as Jun and Fos preferential targets (Handel et al, 1995). Sequences similar to the KCR motif occur in other transcription factors, including NF- $\kappa \mathrm{B}$, which is involved in the transcription of numerous genes (Grilli et al, 1993).

In the maturing neurons of the neonatal mouse brain both $A 7(74)$ and L10 infection can result in apoptotic cell death (Allsopp et al, 1998). In the adult mouse where A7(74) replication is restricted (Pathak and Webb, 1978; Fazakerley et al, 1993; Oliver et al, 1997) and where in the absence of immune responses, virus can persist for long periods in individual scattered cells (Fazakerley and Webb, 1987a; Amor et al, 1996), there is minimal apoptotic death (Fazakerley et al, unpub-

riboprobe) in peripheral tissues: (F) skeletal muscle infected with SFV L10 (PID 4), (G) virus laden pancreas from an SFV A7(74) infected mouse pre-treated with GSTM. Infection was predominantly in acinar cells of the exocrine pancreas (arrows). (H) section of pancreas immunostained for SFV capsid protein (brown) and TUNEL stained for apoptotic nuclei (blue); counterstained with methyl green. 
lished results). An age-related change in neuronal susceptibility to Sindbis virus induced apoptosis has also been observed (Lewis et al, 1996). Despite the increased A7(74) virus load and the increased number of infected cells in the CNS of aurothiolate pretreated (3-4-week-old) mice, infected cells were not TUNEL-positive nor did they show any morphological changes consistent with apoptosis. Thus despite the widespread and productive nature of the A7(74) infection in neurons of the aurothiolate treated mice, these mature neurons remained refractory to apoptotic cell death, at least over the period of the study.

Both GSTM and GSTG altered the pattern of A7(74) infection in peripheral tissues. While skeletal muscle fibres are the major site of L10 infection in the periphery, with discrete foci also observed in myocytes, aurothiolate-enhanced A7(74) replication showed little evidence of skeletal muscle involvement. In aurothiolate treated infected mice, small foci of A7(74) infection were observed in myocytes and rarely, spleen cells but most striking was the heavy infection of pancreatic tissue. This was most pronounced in pancreatic acinar cells. These cells are particularly rich in intracellular membranes and are highly active in terms of intracellular membrane transport. A 500fold enhancement of coxsackievirus B3 titer in the pancreas of GSTM treated mice has previously been reported (Kabiri et al, 1978). Although picornaviruses are not enveloped, their replication is critically dependent on the availability of intracellular smooth membranes and on lipid biosynthesis (Guinea and Carrasco, 1990; Caliguiri and Tamm, 1970). The coxsackie virus data is therefore consistent with gold (I)-enhancement of virus replication operating through effects on intracellular membrane biochemistry.

Gold (I)-induced alterations in intracellular smooth membrane biochemistry seem most likely to play a pivotal role in the enhancement of A7(74) replication. However, the potential for altered gene expression in other systems and, or immunomodulatory effects on soluble mediators such as cytokines and growth factors or NK cells must also be considered. It will be important to carry out further studies to elucidate the exact mechanisms involved and it is hoped this will further illuminate the requirements and restraints for virus infections of mature differentiated neurons.

\section{Materials and methods}

\section{Virus}

The A7(74) and L10 strains of Semliki Forest virus (SFV) were as described previously (Fazakerley et al, 1993). Plaque assays were performed on $80 \%$ confluent BHK cell monolayers in 6-well tissue culture plates (Falcon, Beckton Dickinson Labware) as described previously (Fazakerley et al, 1993).
Mice and gold treatment

Three- to four-week-old, specific pathogen-free, female Balb/c mice were obtained from OLAC (UK). Mice were inoculated ip with $5 \times 10^{3}$ plaque forming units (p.f.u.) of virus, in $0.1 \mathrm{ml}$ phosphate buffered saline (PBS) containing $0.75 \%$ bovine serum albumin. Ten mg doses of sodium aurothiomalate (GSTM; Myocrisin; May \& Baker, England) or aurothioglucose (GSTG; Sigma) were administered ip to 3-4-week-old mice $3-4 \mathrm{~h}$ prior to infection with the A7(74) strain of SFV. Control mice were administered $10 \mathrm{mg}$ of thiomalic acid (TMA; Sigma) ip. GSTM was obtained as a $100 \mathrm{mg} /$ $\mathrm{ml}$ solution and GSTG was dissolved to $100 \mathrm{mg} / \mathrm{ml}$ in sterile distilled water. TMA was dissolved to $100 \mathrm{mg} / \mathrm{ml}$ in sterile distilled water and the $\mathrm{pH}$ adjusted to $6.9-7.2$ with $4.4 \%$ sodium bicarbonate.

\section{Sampling and tissue processing}

Mice were killed by ether anaesthesia. Brains were removed and one half frozen immediately and stored at $-70^{\circ} \mathrm{C}$ for virus titer determination while the other was fixed in $10 \%$ neutral phosphatebuffered formalin.

\section{In situ hybridization}

In situ hybridization was performed on $5 \mu \mathrm{m}$ sagittal sections of paraffin embedded brain sections cut onto poly-L-lysine (Sigma) or Biobond (British BioCell International) coated glass slides. Riboprobes were transcribed in vitro with $\mathrm{T} 7$ polymerase (using a riboprobe Gemini kit; Promega) from HincII linearized pGEM1-SFV (a kind gift from Dr H Garoff, Karolinska Institute, Sweden). As a control, some sections were hybridized with a riboprobe to the P1 region of the unrelated Theiler's virus. Probes were hydrolyzed in $0.04 \mathrm{M} \mathrm{NaHCO}_{3}$ $\left(30 \mathrm{~min}, 60^{\circ} \mathrm{C}\right.$ ) prior to use. In situ hybridization with ${ }^{35}$ S-labelled riboprobes was carried out as detailed previously (Fazakerley et al, 1991, 1993). In situ hybridization with digoxigenin-labelled riboprobes was performed as detailed in Oliver et al (1997).

Autoradiographic images of sections hybridized with ${ }^{35}$ S-labelled riboprobes were produced by exposure of air dried sections to Hyperfilm $\beta$ max (Amersham, UK). The sections were subsequently dipped in photographic emulsion (Amersham, LM1, diluted with $0.66 \mathrm{M} \mathrm{NH}_{4}$ acetate) and exposed, usually for 7 days, at $4^{\circ} \mathrm{C}$. Sections were counterstained with hematoxylin and eosin and examined by bright and dark field microscopy.

\section{Immunostains}

Paraffin embedded tissue sections were dewaxed in xylene, permeabilized with $0.3 \%$ Triton X-100 and proteinase $\mathrm{K}$ treated as detailed previously (Fazakerley et al, 1991, 1993). After blocking with 10\% new-born goat serum, viral proteins were detected in paraffin embedded tissue sections, using a 
monospecific rabbit antipeptide antisera specific for the viral capsid protein; followed by a biotinylated goat anti-rabbit IgG as secondary antibody (Vector Laboratories), an avidin-biotin-peroxidase amplification step (Vector) and diaminobenzidine (Sigma) as substrate.

\section{Terminal deoxynucleotidyltransferase-mediated dUTP nick end-labelling (TUNEL)}

Paraffin embedded tissue sections were dewaxed in xylene and permeabilized with Triton X-100 and proteinase $\mathrm{K}$. One specimen was designated as a positive control, equilibrated with DNase 1 buffer (30 mM Tris $\mathrm{pH} 7.2 ; 140 \mathrm{mM}$ sodium cacodylate; $4 \mathrm{mM} \mathrm{MgCl}_{2} ; 0.1 \mathrm{mM}$ dithiothreitol) for $20 \mathrm{~min}$ and then incubated in this buffer with $5 \mu \mathrm{g} / \mathrm{ml}$ DNase 1 at $37^{\circ} \mathrm{C}$ for $30 \mathrm{~min}$. After permeabilization the remaining specimens were rinsed with PBS and equilibrated with TUNEL buffer $(30 \mathrm{mM}$ Tris pH 7.2; $140 \mathrm{mM}$ sodium cacodylate; $1 \mathrm{mM}$ cobalt chloride) for $20 \mathrm{~min}$. All specimens were incubated with $0.3 \mathrm{U} / \mu$ l terminal deoxynucleotidlytransferase (TdT); $20 \mu \mathrm{M}$ Digoxigenin-11-dUTP; $30 \mathrm{mM}$ Tris $\mathrm{pH} 7.2 ; 140 \mathrm{mM}$ sodium cacodylate and $1 \mathrm{mM}$ cobalt chloride for $2 \mathrm{~h}$ at $37^{\circ} \mathrm{C}$ in a humidified container. As a negative control TdT enzyme was omitted from one reaction. The reaction was terminated with $2 \times$ SSC for 5 min at room temperature. Incorporated digoxigenin-dUTP was detected

\section{References}

Allner K, Bradish CJ, Fitzgeorge R, Nathanson N (1974). Modifications by sodium aurothiomalate of the expression of virulence in mice by defined strains of Semliki Forest virus. J Gen Virol 24: 221-228.

Allsopp TE, Scallan MF, Williams A, Fazakerley JK (1998). Virus infection induces neuronal apoptosis: A comparison with trophic factor withdrawal. Cell Death Diff 5: 50-59.

Amor S, Scallan MF, Morris MM, Dyson H, Fazakerley JK (1996). Role of immune responses in protection and pathogenesis during Semliki Forest virus encephalitis. J Gen Virol 77: 281-291.

Braas KM, Newby AC, Wilson VS, Synder SH (1986). Adenosine-containing neurons in the brain localized by immunocytochemistry. J Neurol Sci 6: 1952-1961.

Bradish CJ, Allner K, Maber HB (1971). The virulence of original and derived strains of Semliki Forest virus for mice, guinea-pigs and rabbits. J Gen Virol 12: $141-$ 160.

Bradish CJ, Allner K, Fitzgeorge R (1975). Immunomodification and the expression of virulence in mice by defined strains of Semliki Forest virus: the effects of cyclophosphamide. J Gen Virol 28: $225-237$.

Caliguiri LA, Tamm I (1970). The role of cytoplasmic membranes in poliovirus biosynthesis. Virology 4: $100-111$.

Dailey ME, Bridgman PC (1993). Vacuole dynamics in growth cones: correlated EM and video observations. J Neurosci 13: 3375-3393. by enzyme-linked immunoassay, using an antidigoxigenin alkaline phosphatase conjugate (antiDIG-AP) to catalyze a colour reaction between 5bromo-4-chloro-3-indoyl phosphate (X-phosphate) and nitroblue tetrazolium salt (NBT) which deposits a blue precipitate. Specimens were equilibrated with PBS, blocked with $3 \%$ normal goat serum (NGS) for $30 \mathrm{~min}$, washed in PBS-T $(0.05 \%$ Tween $20 ; 2 \times 5 \mathrm{~min}$ ) and incubated with anti-DIG-AP (Boehringer) diluted 1:100 in PBS with 1\% NGS. The specimens were then washed with PBS $(3 \times 5 \mathrm{~min})$ and incubated with NBT and X-phosphate solutions as detailed in the Boehringer Detection Kit (cat. no. 1175 041). Immunostains for SFV capsid proteins were performed after the TUNEL protocol, as detailed above. Sections were counter stained with methyl green at $60^{\circ} \mathrm{C}$ for $10 \mathrm{~min}$, rinsed in $95 \%$ alcohol followed by immersion in $100 \%$ alcohol $(2 \times 1 \mathrm{~min})$. Each slide was placed in Histoclear $(2 \times 15 \mathrm{~min})$ and mounted in Surgimount mounting medium (Surgipath).

\section{Acknowledgements}

This research was supported by a grant from the UK Medical Research Council. We are grateful to Heather Dyson for excellent technical assistance.

Fazakerley JK, Webb HE (1987a). Semliki Forest virusinduced, immune-mediated demyelination - adoptive transfer studies and viral persistence in nude-mice. $J$ Gen Virol 68: 377-385.

Fazakerley JK, Webb HE (1987b). Semliki Forest virus induced, immune mediated demyelination: the effect of irradiation. Br J Exp Pathol 68: 101-113.

Fazakerley JK, Southern P, Bloom F, Buchmeier MJ (1991). High-resolution in situ hybridization to determine the cellular distribution of lymphocytic choriomeningitis virus RNA in the tissues of persistently infected mice - relevance to arenavirus disease and mechanisms of viral persistence. J Gen virol 72: $1611-1625$.

Fazakerley JK, Pathak S, Scallan M, Amor S, Dyson H (1993). Replication of the A7(74) Strain of Semliki Forest virus is restricted in neurons. Virology 195: $627-637$.

Froshauer S, Kartenbeck J, Helenius A (1988). Alphavirus RNA replicase is located on the cytoplasmic surface of endosomes and lysosomes. J Cell Biol 107: $2075-2086$.

Gates MC, Sheahan BJ, Atkins GJ (1984). The pathogenicity of the M9 mutant of Semliki Forest virus in immune-compromised mice. J Gen Virol 65: 73-80. 
Griem P, Takahashi K, Kalbacher H, Gleichmann E (1995). The antirheumatic drug disodium aurothiomalate inhibits $\mathrm{CD} 4+\mathrm{T}$ cell recognition of peptides containing two or more cysteine residues. I Immunol 155: $1575-1587$.

Grilli M, Chiu JJ, Lenardo MJ (1993). NF-kappa B and Rel: participants in a multiform transcriptional regulatory system. Inter Rev Cytol 143: 1-62.

Guinea R, Carrasco L (1990). Phospholipid biosynthesis and poliovirus genome replication, two coupled phenomena. EMBO J 9: 2011-2016.

Handel ML, Watts CKW, Defazio A, Day RO, Sutherland RL (1995). Inhibition of Ap-1 binding and transcription by gold and selenium involving conserved cysteine residues in Jun and Fos. PNAS (US) 92: $4497-4501$.

Kabiri M, Basiri E, Kadivar D (1978). Potentiation of coxsackievirus B3 infection in adult mice pretreated with a gold salt. J Med Virol 3: 125-136.

Koike R, Miki I, Otoshi M, Totsuka T, Inoue H, Kase H, Saito I, Miyasaka N (1994). Gold sodium thiomalate down-regulates intercellular adhesion molecule-1 and vascular cell adhesion molecule-1 expression on vascular endothelial cells. Molec Pharmacol 46: $599-604$.

Kuge O, Akamatsu Y, Nishijima M (1989). Abortive infection with Sindbis virus of a Chinese hamster ovary cell mutant defective in phosphatidylserine and phosphatidylethanolamine biosynthesis. Biochim Biophys Acta 989: 61-69.

Lawson KJ, Danpure CJ, Fye DA (1977). The uptake and subcellular distribution of gold in rat liver cells after in vivo administration of sodium aurothiomalate. Biochem Pharmacol 26: 2417-2426.

Lewis J, Wesselingh SL, Griffin DE, Hardwick JM (1996). Alphavirus induced apoptosis in mouse brains correlates with neurovirulence. J Virol 70: 1828-1835.

McIntosh BM, Brookworth C, Kokernot RH (1961). Isolation of Semliki Forest virus from Aedes (Aedimorphus) argenteopunctatus (THEOBALD) collected in Portuguese East Africa. Trans Roy Soc Trop Med Hyg 55: 192.

Mehta S, Webb HE (1982). Lysosomal enzyme changes in macrophages from mice given myocrisin and infected with avirulent Semliki Forest virus. Br J Exp Path 63: $443-446$.

Mehta S, Webb HE (1987). The effect of gold sodium thiomalate in adult Swiss/A2G mice infected with Togaviruses and Flaviviruses. J Gen Virol 68: 26652668.

Mehta S, Webb HE (1990). The effect of gold sodium thiomalate and its constituent part, thiomalate, on the virus titres and the lysosomal enzyme activity in peritoneal macrophages of Swiss/A2G mice infected with the avirulent strain of Semliki Forest virus. J Exp Pathol (Oxford) 71: 359-366.

Mehta S, Pathak S, Webb HE (1990). Induction of membrane proliferation in mouse CNS by gold sodium thiomalate with reference to increased virulence of the avirulent Semliki Forest virus. Biosci Rep 10: $271-279$.
Oliver KR, Scallan MF, Dyson H, Fazakerley JK (1997). Susceptibility to a neurotropic virus and its changing distribution in the developing brain is a function of CNS maturity. J Neurovirol 3: $38-48$.

Oliver KR, Fazakerley JK (1998). Transneuronal spread of Semliki Forest virus in the developing mouse olfactory system is determined by neuronal maturity. Neurosci 82: 867-877.

Pathak S, Webb HE (1974). Possible mechanisms for the transport of Semliki Forest virus into and within mouse brain: An electron microscopic study. J Neurol Sci 23: $175-184$.

Pathak S, Webb HE (1978). An electron-microscopic study of avirulent and virulent Semliki Forest virus in the brains of different ages of mice. J Neurol Sci 39: $199-211$

Pathak S, Webb HE (1980). The entry and the transport of arboviruses into and throughout mouse brain: An electron-microscopic study. Electron Microscopy 2: $492-493$.

Pathak S, Webb HE (1983). Effect of myocrisin (sodium auro-thio-malate) on the morphogenesis of avirulent Semliki Forest virus in mouse brain: An electron microscopical study. Neuropath Appl Neurobiol 9: $313-327$

Pathak S, Webb HE (1988a). An electron microscopical study of the replication of avirulent Semliki Forest virus in the retina of mice. J Neurol Sci 85: 87-96.

Pathak S, Webb HE (1988b). Cytoplasmic viral core aggregates and budding of mature virus and spherules in mouse brain following Semliki Forest virus infections. Inst Phys Conf Ser 3: 213-214.

Peranen J, Laakkonen P, Hyvonen M, Kaariainen L (1995). The alphavirus replicase protein nsp1 is membrane-associated and has affinity to endocytic organelles. Virology 208: 610-620.

Perez L, Guinea R, Carrasco L (1991). Synthesis of Semliki Forest virus RNA requires continuous lipid synthesis. Virology 183: 74-82.

Pusztai R, Gould E, Smith H (1971). Infection pattern in mice of an avirulent and virulent strain of Semliki Forest virus. Br J Exp Pathol 52: 669-677.

Smithburn KC, Haddow WJ (1944). Semliki Forest virus. I. Isolation and pathogenic properties. J Immunol 49: $141-145$.

Subak-Sharpe I, Dyson H, Fazakerley J (1993). In-vivo depletion of CD8+ T-cells prevents lesions of demyelination in Semliki Forest virus-infection. J Virol 67: $7629-7633$

Suckling AJ, Pathak S, Jagelman S, Webb HE (1978). Virus associated demyelination: a model using avirulent Semliki Forest virus infection of mice. J Neurol Sci 36: $147-154$. 